\title{
Artificial Collimation of Fast-Electron Beams with Two Laser Pulses
}

\author{
A. P. L. Robinson, ${ }^{1}$ M. Sherlock, ${ }^{1,2}$ and P. A. Norreys ${ }^{1,3}$ \\ ${ }^{1}$ Central Laser Facility, STFC Rutherford-Appleton Laboratory, Chilton, Didcot, Oxfordshire, OX11 OQX, United Kingdom \\ ${ }^{2}$ SUP A, Department of Physics, University of Strathclyde, Glasgow, G4 ONG, United Kingdom \\ ${ }^{3}$ Plasma Physics Group, Blackett Laboratory, Imperial College London, SW7 2BZ, United Kingdom
}

(Received 8 May 2007; published 17 January 2008)

\begin{abstract}
A scheme for artificially collimating fast-electron beams produced in high intensity $\left(>10^{19} \mathrm{~W} \mathrm{~cm}^{-2}\right)$ laser-solid interactions is proposed. The scheme uses a laser pulse at the relativistic threshold $\left(\approx 10^{18} \mathrm{~W} \mathrm{~cm}^{-2}\right)$ that precedes the high intensity pulse to pregenerate a collimating magnetic field. This concept is supported by analytical calculations and numerical calculations performed using a novel hybrid-Vlasov-Fokker-Planck code called LEDA. This scheme may be highly useful for fast ignition inertial confinement fusion.
\end{abstract}

DOI: 10.1103/PhysRevLett.100.025002

PACS numbers: 52.38. $-\mathrm{r}$, 52.25.Dg, 52.57.Kk, 52.65.Ff

When a laser pulse with an irradiance in excess of $10^{18} \mathrm{~W} \mathrm{~cm}^{-2}$ interacts with an overdense plasma a considerable fraction of the laser energy is absorbed as "fast" electrons, which are nonthermal, relativistic electrons $(>100 \mathrm{keV})$. There has been intense interest in the fast electrons since a fast-electron beam can, in principle, heat compressed fuel to ignition temperatures in an inertial confinement fusion (ICF) target in a scheme known as fast ignition ICF [1]. Additionally the fast electrons can accelerate ions as the fast electrons attempt to escape the target and are confined by space charge [2-4]. This has led to extensive experimental [5-10] and theoretical [11-15] studies of fast-electron transport in solids and dense plasmas.

Until now very few ideas have been proposed regarding the control of the fast-electron beam, particularly controlling the transverse extent (divergence or collimation) of the beam. This is important, as it determines the overall efficiency of most applications, especially fast ignition. The approach proposed by Campbell et al. [16] exploits the space-charge confinement at plasma-vacuum interfaces. In that work, it was demonstrated that within a "plug" of material surrounded by vacuum gaps the fast electrons would be confined. However, in the case of fast ignition, the use of the collimator complicates matters, as it must be ensured that the vacuum gaps are not compromised during the compression of the deuterium-tritium fuel. A collimation scheme that is not dependent on target engineering will be a significant step, not only in fast ignition ICF, but in most aspects of high intensity laser-solid interactions.

In this Letter, a completely new scheme that produces a collimated fast-electron beam in a homogeneous target is proposed. This utilizes two successive laser pulses that are focused to the same spot. The first (generator) pulse is a $10^{18} \mathrm{~W} \mathrm{~cm}^{-2}$ pulse that pregenerates a collimating magnetic field, and the second (main) is a high intensity $>10^{19} \mathrm{~W} \mathrm{~cm}^{-2}$ pulse that produces a population of $\mathrm{MeV}$ fast electrons. Even when the $\mathrm{MeV}$ fast electrons enter the target with an extremely large divergence angle, they are collimated into a tight beam.

First the basic analytical considerations are discussed, after which the results of detailed numerical calculations are reported. The numerical calculations were performed using a novel 2D hybrid-Vlasov-Fokker-Planck code called LEDA.

Previously Bell and Kingham considered the "natural" resistive collimation of a fast-electron beam by its own resistively generated magnetic field [17]. Importantly, "natural collimation" was shown to be less favorable at high divergence angle, laser power, and fast-electron temperature. This makes natural collimation unfavorable at intensities in excess of $10^{19} \mathrm{~W} \mathrm{~cm}^{-2}$.

Suppose that an azimuthal magnetic field was pregenerated, with flux density $B_{0}$ and a spatial extent $L_{x}$ in the $x$ direction (into the target parallel to the target normal), and $L_{y}$ in the $y$ direction (parallel to target surface). Let the generator pulse have duration $t_{\mathrm{gen}}$, and the fast electrons produced by the generator pulse have speed $v_{\text {gen }}$. The laser spot of the generator pulse has radius $R_{\text {gen }}$ and its fastelectron current density is $j_{\text {gen }}$. Let the fast-electron beam that is produced by the main pulse have a divergence halfangle of $\theta_{1 / 2}$ (in radians), and the fast-electron speed and Lorentz factor be $v_{f}$ and $\gamma_{f}$, respectively. Throughout we assume that $R_{\text {gen }} \geq R_{\text {main }}$. Collimation should occur when the angle of deflection of a fast electron during its transit through the pregenerated magnetic field is roughly equal to the divergence half-angle of the beam. By considering the trajectory of a fast electron that enters at the divergence angle one then concludes that

$$
\begin{gathered}
B_{0} L_{y}>\frac{\gamma_{f} v_{f} m_{e}}{e}\left(1-\cos \theta_{1 / 2}\right), \quad \text { and } \\
B_{0} L_{x}>\frac{\gamma_{f} v_{f} m_{e}}{e} \sin \theta_{1 / 2},
\end{gathered}
$$

must be satisfied. 
Generally one expects that $L_{x}>L_{y}$ (and that $L_{y} \approx$ $R_{\text {gen }}$ ); therefore Eq. (1) should be taken as the primary condition for artificial collimation. One can estimate $B_{0} L_{y}$ from the generator pulse parameters. Using the approximation that $\partial B_{0} / \partial t \approx \eta j_{\text {gen }} / R_{\text {gen }}$, where $\eta$ is an average target resistivity, one estimates that $B_{0} \approx \eta j_{\text {gen }} t_{\text {gen }} / R_{\text {gen }}$. On substituting this into Eq. (1), and replacing $\gamma_{f} v_{f}$ by the ponderomotive scaling [18], one obtains a single, primary condition for artificial collimation:

$$
\begin{aligned}
\eta j_{\text {gen }} t_{\text {gen }}> & \frac{m_{e} c}{e} \sqrt{1+\frac{I \lambda^{2}}{1.38 \times 10^{18} \mathrm{~W} \mathrm{~cm}^{-2} \mu \mathrm{m}^{2}}} \\
& \times\left(1-\cos \theta_{1 / 2}\right)
\end{aligned}
$$

where $j_{\text {gen }} \approx \frac{\beta I_{\text {gen }}}{2 \times 10^{5}\left(I_{\text {gen }} \lambda^{2} / 10^{18} \mathrm{~W} \mathrm{~cm}^{-2} \mu \mathrm{m}^{2}\right)^{1 / 3}}$

In the above expression $\beta$ is the laser conversion efficiency for the generator pulse. The target resistivity accounts for the target composition and initial temperature. Taking $\eta=8 \times 10^{-7} \Omega \mathrm{m}$ (i.e., assume average resistivity equivalent to $\mathrm{Al}$ at $300 \mathrm{eV}), j_{\text {gen }}=10^{16} \mathrm{~A} \mathrm{~m}^{-2}, t_{\text {gen }}=$ $500 \mathrm{fs}, \theta_{1 / 2}=\pi / 4$, and $I \lambda^{2}=5 \times 10^{19} \mathrm{~W} \mathrm{~cm}^{2} \mu \mathrm{m}^{2}$, one finds that Eq. (3) is satisfied. Note that this also predicts that the generator pulse limits the main pulse intensity. If one were to change these parameters such that $I \lambda^{2}>1 \times 10^{20} \mathrm{~W} \mathrm{~cm}^{2} \mu \mathrm{m}^{2}$, then Eq. (3) would no longer be satisfied.

Therefore one expects the pregenerated field to collimate the fast-electron beam produced by the main pulse, for this set of parameters. However, this simple analytic approach cannot encompass all of the relevant physics. Therefore the use of sophisticated numerical calculations to further study the scheme and to test the validity of Eq. (3) is required.

The case that has been studied numerically is that of a homogeneous solid foil which is irradiated by the two pulses on one side. This is primarily relevant to current laboratory experiments. The LEDA code which has been used to study this problem is a 2D hybrid Vlasov-FokkerPlanck (VFP) code that can be thought of as a synthesis of the KALOS code of Bell and Kingham [17] and the hybrid code of Davies [19]. Specifically the fast electrons are treated via a KALOS-like algorithm. The distribution function of the fast electrons is expressed as a (truncated) spherical harmonic expansion with terms of the form $f_{l}^{m}(x, y, p) P_{l}^{m}(\cos \theta) \exp (i m \phi)$. Substituting this expansion into the VFP equation yields a set of equations for the $f_{l}^{m}(x, y, p)$ coefficients, and it is these equations which the code solves. The KALOS algorithm is explained in some detail in [20]. LEDA utilizes a robust characteristic based method for the $x$ advection that was previously employed in the code in [21], while Runge-Kutta methods are used for $p$ advection and $y$ advection. All of the spatial boundaries are reflective.
The hybrid treatment of the background (an Al target was modeled) uses the same fit to Milchberg's work [22] for the resistivity and the same Thomas-Fermi model for the specific heat capacity (SHC) that Davies uses [19]. The electric field is determined by the simple Ohm's law, $\mathbf{E}=$ $-\eta \mathbf{j}_{f}$, and the magnetic field from $\partial \mathbf{B} / \partial t=-\boldsymbol{\nabla} \times \mathbf{E}$. The background temperature evolves only due to Ohmic heating. The fast electrons are injected in the first spatial cell on the left-hand end of the grid, and the "laser" intensity is modeled by $I=\beta I_{0} \exp \left[-\left(y-y_{\max } / 2\right)^{2} / R^{2}\right]$ (constant over the injection time, $t_{\text {pulse }}$ ). The fast-electron temperature, $T_{f}$, is determined by Beg's law [5] for $I_{0}<$ $10^{19} \mathrm{~W} \mathrm{~cm}^{-2}$, and by the scaling law of Wilks and Kruer [18] for $I_{0}>10^{19} \mathrm{~W} \mathrm{~cm}^{-2}$. The injected fast-electron distribution function is specified to be $f_{\text {injected }} \propto$ $\left(\cos ^{M} \theta\right) p^{2} \exp \left(-\sqrt{p^{2}+m_{e}^{2} c^{2}} / k_{B} T f\right)$. This distribution is a relativistic Maxwellian which is beamed into the target with a divergence half-angle of $\approx 67^{\circ} / \sqrt{M}$.

In the standard run a uniform spatial grid with 140 cells in $x$ and $y$ and a cell size of $0.75 \mu \mathrm{m}$ was used. The momentum grid used 75 grid points, and 22 harmonics were used in the expansion. The generator pulse was modeled by setting $I_{0}=2.5 \times 10^{18} \mathrm{~W} \mathrm{~cm}^{-2}$ and $t_{\text {pulse }}=$ $500 \mathrm{fs}$, and this was immediately followed by the main pulse, which was modeled by setting $I_{0}=5 \times$ $10^{19} \mathrm{~W} \mathrm{~cm}^{-2}$ and $t_{\text {pulse }}=500 \mathrm{fs}$. For the generator pulse the divergence angle was set by $M=8\left(\approx 24^{\circ}\right.$ half-angle $)$, and for the main pulse the divergence angle was set by $M=2\left(\approx 47^{\circ}\right.$ half-angle). For both pulses, the laser absorption efficiency, $\beta$, was set to 0.3 . The initial background temperature was set to $1 \mathrm{eV}$.

The results of the standard run at $900 \mathrm{fs}$ were compared to runs with the main pulse only at $400 \mathrm{fs}$. It was found that in the standard run the fast electrons were indeed collimated into a well defined beam across the entire computational domain; in contrast, in the comparison runs the fast electrons were weakly collimated at best, or divergent.

This is illustrated by both Figs. 1 and 2. Figure 1 shows plots of the fast-electron density and the magnetic flux density, $B_{z}$. It is clearly seen from Fig. 1 that in the case of the standard run [(a) and (c)] the fast electrons are collimated, whereas in a run with only the main pulse the fast-electron beam diverges [(b) and (d)].

Another comparison is shown in Fig. 2 in which lineouts of the fast-electron density are shown for the standard run and the run with only the main pulse. The reader may have noticed that the fast-electron density in the standard run consists of fast electrons produced by both the generator and the main pulse. For this reason the lineout for a run with only the generator pulse is included to indicate that the fast electrons in the lineout of the standard run are almost all due to the main pulse.

The artificial collimation is found to be caused primarily by the pregenerated magnetic field, although significant generation of collimating magnetic field by the main pulse 
(a)

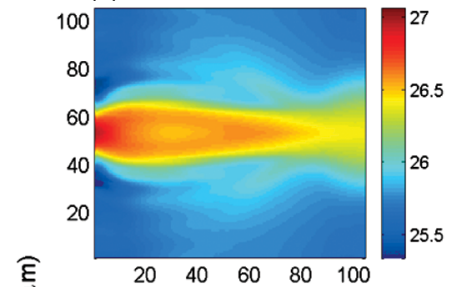

$\stackrel{\frac{E}{3}}{>}$

(c) (b)

(d)

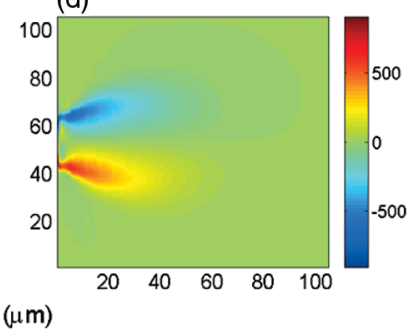

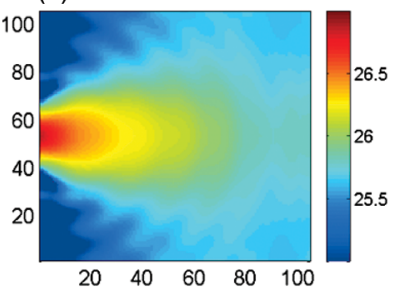

FIG. 1 (color). $\quad \log _{10}$ of fast-electron density $\left(\mathrm{m}^{-3}\right)$ at $900 \mathrm{fs}$ in the standard run (a) and a run with only the main pulse at $400 \mathrm{fs}$ (b). The magnetic flux density, $B_{z}(T)$, that corresponds to (a) is shown in (c) and that of (b) in (d).

of fast electrons does appear to occur and this is responsible for sustaining the collimation of the fast electrons deep into the target. This is shown in Fig. 3 which shows the magnetic flux density in the upper half of the target at various times. This shows that the magnetic flux density and spatial extent at $500 \mathrm{fs}$ are sufficient to cause good collimation, in agreement with Eq. (3) which should be satisfied for these simulation parameters.

In order to test the scheme's robustness and the validity of Eq. (3), further simulations were performed (labeled i to xi) in which particular parameters were changed, while the other parameters were identical to those in the standard run. The divergence angle of the generator beam was increased by setting $M=4$ in one run (i) and $M=2$ in

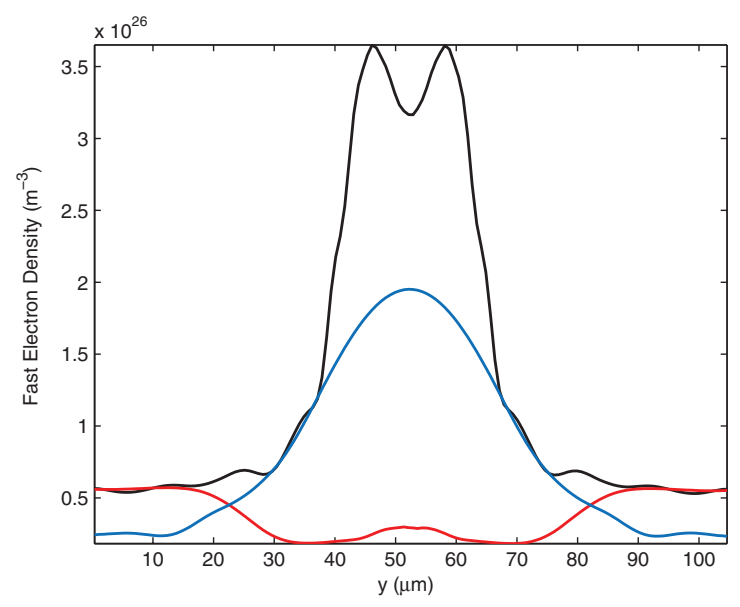

FIG. 2 (color). Graph of fast-electron densities at $x=30 \mu \mathrm{m}$. Black curve: $900 \mathrm{fs}$ in standard run. Blue curve: $400 \mathrm{fs}$ in run with only main pulse. Red curve: 400 fs with only generator pulse. another (ii). In both $\mathrm{i}$ and ii artificial collimation still occurred, as was the case in three runs in which the laser intensity of the generator was set at $7.5 \times 10^{18} \mathrm{~W} \mathrm{~cm}^{-2}$ (iii), $5 \times 10^{18} \mathrm{~W} \mathrm{~cm}^{-2}$ (iv), and $1 \times 10^{18} \mathrm{~W} \mathrm{~cm}^{-2}(\mathrm{v})$.

The capabilities of the pregenerated field in the standard run were tested by carrying out a run in which the main pulse was injected isotropically (vi), and runs in which the intensity of the main pulse was set at $7.5 \times 10^{19} \mathrm{~W} \mathrm{~cm}^{-2}$ (vii), $1 \times 10^{20} \mathrm{~W} \mathrm{~cm}^{-2}$ (viii), and $2 \times 10^{20} \mathrm{~W} \mathrm{~cm}^{-2}$ (ix). In runs vii and viii artificial collimation occurred. In runs vi and ix artificial collimation also occurred, but more of the fast electrons generated by the main pulse escaped laterally than in other runs. Note that the onset of the failure of artificial collimation observed in runs vi and ix is in agreement with Eq. (3) and thus confirms the main pulse intensity and divergence angle limitations that are inherent in Eq. (3).

The conclusion that was drawn from runs $\mathrm{i}-\mathrm{ix}$ was that the artificial collimation that occurred in the standard run was not a special case and that the scheme is not highly sensitive to the initial conditions.

The application of this scheme to fast ignition ICF goes beyond the scope of this Letter. Nonetheless, two points are addressed. First, the standard run shows that artificial collimation occurs for a generator pulse that has $5 \%$ of the energy of the main pulse, and run $v$ shows that it occurs for a generator pulse with $1 \%$ of the energy of the main pulse. Therefore, the scheme does not demand a significant increase in the energy requirement.

The second point concerns the initial electron temperature. In a fast ignition scenario the imploded fuel will be at a temperature of a few hundred eV, not $1 \mathrm{eV}$. Since this implies lower resistivity, one expects magnetic field generation to be weaker, and thus the potential for artificial (a)

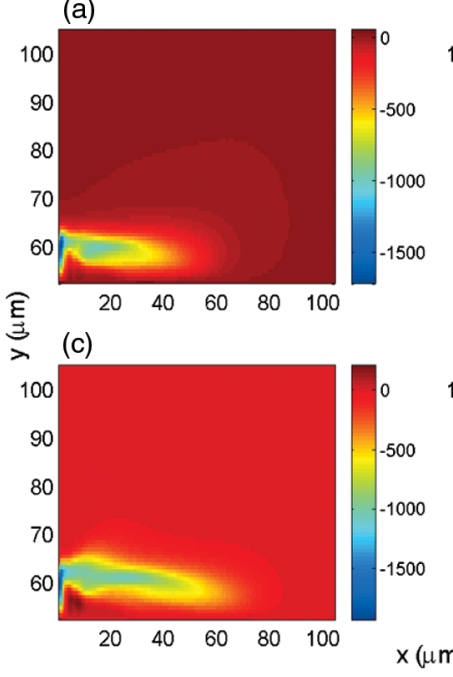

(b)

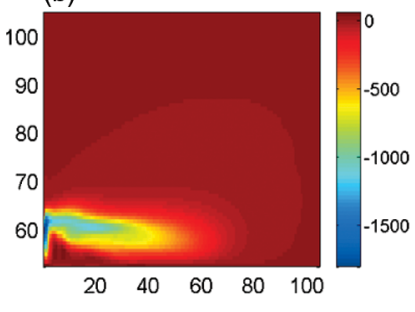

(d)

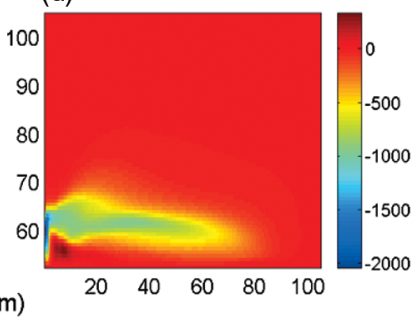

FIG. 3 (color). Magnetic flux density, $B_{z}(T)$, in the upper half $(y>50 \mu \mathrm{m})$ plane at $500 \mathrm{fs}(\mathrm{a}), 600 \mathrm{fs}(\mathrm{b}), 700 \mathrm{fs}(\mathrm{c})$, and $800 \mathrm{fs}$ (d), in the standard run. 
(a)
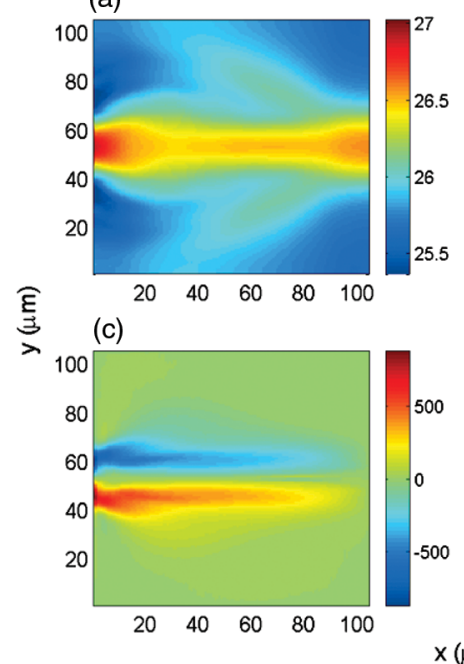

(b)

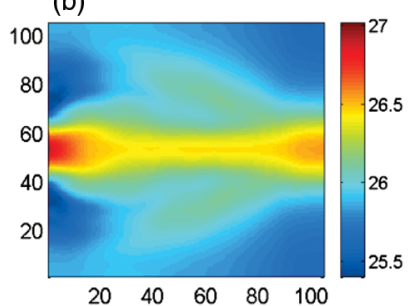

(d)

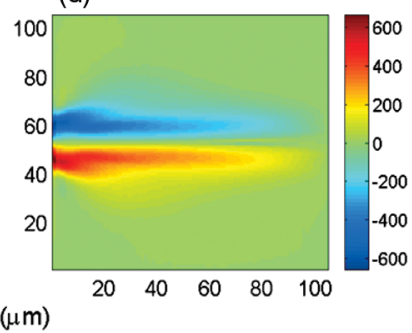

FIG. 4 (color). $\quad \log _{10}$ fast-electron density $\left(\mathrm{m}^{-3}\right)$ in run $\mathrm{x}(\mathrm{a})$ and run xi (b), and $B_{z}(T)$ in run x (c) and run xi (d) all at $900 \mathrm{fs}$.

collimation. Nonetheless, the case that satisfies Eq. (3) assumed an average target temperature of $300 \mathrm{eV}$, so artificial collimation may still occur. This concern was investigated by carrying out two further runs, $\mathrm{x}$ and $\mathrm{xi}$, which were identical to the standard run except that the initial background temperatures were set to $200 \mathrm{eV}$ and $300 \mathrm{eV}$, respectively. The results of these runs are shown in Fig. 4.

It can be seen by comparing Figs. 1 and 4 that the degree of collimation in runs $\mathrm{x}$ and $\mathrm{xi}$ is considerably better than it is in the standard run. When main pulse only runs are carried out at initial temperatures of 200 and $300 \mathrm{eV}$, the results are very similar to those at $1 \mathrm{eV}$, i.e., no collimation. Although Eq. (3) predicts artificial collimation in runs $\mathrm{x}$ and xi, it does not predict an improvement over the standard run. This occurs because the collimation process in the standard run is compromised to a certain extent by an occurrence of Davies' "beam-hollowing" process [23], which does not occur in runs $\mathrm{x}$ and $\mathrm{xi}$. This can be seen in Figs. 1(a) and 1(c). Semianalytic calculations were performed using a modified version of Davies "rigid beam" model that includes the resistivity and SHC models used in LEDA instead of the $\eta \propto T^{\alpha}$ and constant SHC used in [23]. It was found that, at $500 \mathrm{fs}, B_{\phi}(r)<0(\forall r)$ in the case of a target initially at $200 \mathrm{eV}$, i.e., no beam hollowing. Whereas in the case of a $1 \mathrm{eV}$ initial temperature, $B_{\phi}>0$ in a small region close to the axis, i.e., beam hollowing. Therefore the improvement in collimation is actually due to a suppression of beam hollowing, and this is predicted by semianalytic models that go beyond the arguments that were stated earlier in this Letter. Only when the initial temperature exceeds $1 \mathrm{keV}$ does the pregenerated magnetic field become sufficiently weak to prevent artificial collimation; this is in agreement with Eq. (3).

In summary, a scheme for artificially inducing the collimation of fast electrons, even when the initial divergence angle of the beam is large, has been proposed. The scheme utilizes two laser pulses-a generator pulse of $I \approx$ $10^{18} \mathrm{~W} \mathrm{~cm}^{-2}$ which generates a collimating magnetic field, and a main pulse $I>10^{19} \mathrm{~W} \mathrm{~cm}^{-2}$ which produces the fast-electron beam to be collimated. An analytical condition for artificial collimation was derived, and numerical calculations performed using the new LEDA hybrid-VFP code have demonstrated that artificial collimation occurs. Furthermore, LEDA calculations performed using a range of initial conditions have shown that the scheme is robust. The scheme should be well suited to fast ignition ICF as it requires only a small increase $(<10 \%)$ in the short-pulse energy requirement. It is envisaged that this scheme will find applications across the entire spectrum of high intensity laser-plasma research.

The authors would like to thank J.R. Davies for his insights and fruitful discussions. The authors are also grateful for the use of computing resources provided by STFC's $e$-Science facility.

[1] M. Tabak et al., Phys. Plasmas 1, 1626 (1994).

[2] R. A. Snavely et al., Phys. Rev. Lett. 85, 2945 (2000).

[3] E. L. Clark et al., Phys. Rev. Lett. 84, 670 (2000).

[4] T. Cowan et al., Phys. Rev. Lett. 92, 204801 (2004).

[5] F. N. Beg et al., Phys. Plasmas 4, 447 (1997).

[6] J. Fuchs et al., Phys. Rev. Lett. 91, 255002 (2003).

[7] R. Stephens et al., Phys. Rev. E 69, 066414 (2004).

[8] E. Martinolli, Phys. Rev. E 73, 046402 (2006).

[9] K. Lancaster et al., Phys. Rev. Lett. 98, 125002 (2007).

[10] K. Tanaka et al., Phys. Plasmas 7, 2014 (2000).

[11] J. R. Davies, Phys. Rev. E 68, 056404 (2003).

[12] R. J. Mason, Phys. Rev. Lett. 96, 035001 (2006).

[13] R. G. Evans et al., Appl. Phys. Lett. 86, 191505 (2005).

[14] J. J. Honrubia et al., Laser Part. Beams 22, 129 (2004).

[15] T. Taguchi et al., Phys. Rev. Lett. 86, 5055 (2001).

[16] R. B. Campbell et al., Phys. Plasmas 10, 4169 (2003).

[17] A. R. Bell and R. J. Kingham, Phys. Rev. Lett. 91, 035003 (2003).

[18] S. C. Wilks and W.L. Kruer, IEEE J. Quantum Electron. 33, 1954 (1997).

[19] J. R. Davies, Phys. Rev. E 65, 026407 (2002).

[20] A. R. Bell et al., Plasma Phys. Controlled Fusion 48, R37 (2006).

[21] A. P. L. Robinson et al., Plasma Phys. Controlled Fusion 48, 1063 (2006).

[22] H. M. Milchberg et al., Phys. Rev. Lett. 61, 2364 (1988).

[23] J. R. Davies et al., Plasma Phys. Controlled Fusion 48, 1181 (2006). 